OPEN ACCESS

Edited by:

Snehal Rajendra Jadhav,

Deakin University, Australia

Reviewed by:

Xuetong Fan,

Agricultural Research Service,

United States

Achyut Adhikari,

Louisiana State University,

United States

*Correspondence:

Thomas M. Taylor

matt_taylor@tamu.edu

†These authors have contributed equally to this work

¥Present address:

Keila L. Perez-Lewis, International Grains and Cereal, LLC

Dallas, TX, United States

Specialty section: This article was submitted to

Agro-Food Safety,

a section of the journal

Frontiers in Sustainable Food Systems

Received: 28 December 2020 Accepted: 11 March 2021

Published: 09 April 2021

Citation:

Yegin Y, Perez-Lewis KL, Liu S, Kerth CR, Cisneros-Zevallos $L$,

Castillo A, Akbulut M and Taylor TM

(2021) Antimicrobial-Loaded

Polymeric Micelles Inhibit Enteric

Bacterial Pathogens on Spinach Leaf

Surfaces During Multiple Simulated

Pathogen Contamination Events.

Front. Sustain. Food Syst. 5:646980.

doi: 10.3389/fsufs.2021.646980

\section{Antimicrobial-Loaded Polymeric Micelles Inhibit Enteric Bacterial Pathogens on Spinach Leaf Surfaces During Multiple Simulated Pathogen Contamination Events}

\author{
Yagmur Yegin ${ }^{1 \dagger}$, Keila L. Perez-Lewis ${ }^{1+\neq}$, Shuhao Liu ${ }^{2}$, Chris R. Kerth ${ }^{3}$, \\ Luis Cisneros-Zevallos ${ }^{4}$, Alejandro Castillo ${ }^{1}$, Mustafa Akbulut ${ }^{2}$ and Thomas M. Taylor ${ }^{3 *}$

\begin{abstract}
${ }^{1}$ Department of Food Science and Technology, Texas A\&M University, College Station, TX, United States, ${ }^{2}$ Artie McFerrin Department of Chemical Engineering, Texas A\&M University, College Station, TX, United States, ${ }^{3}$ Department of Animal Science, Texas A\&M University, College Station, TX, United States, ${ }^{4}$ Department of Horticultural Sciences, Texas A\&M
\end{abstract} \\ University, College Station, TX, United States
}

Protecting fresh-packed produce microbiological safety against pre- and post-harvest microbial pathogen contamination requires innovative antimicrobial strategies. Although largely ignored in the scientific literature, there exists the potential for gross failure in food safety protection of fresh fruits and vegetables leading to opportunity for multiple produce contamination events to occur during production and post-harvest handling of food crops. The primary objective of this research was to determine the efficacy of plant-derived antimicrobial-loaded nanoparticles to reduce Escherichia coli 0157:H7 and Salmonella enterica serovar Typhimurium on spinach leaf surfaces whilst simulating multiple pathogen contamination events (pre-harvest and post-harvest). Spinach samples were inoculated with a blend of E. coli O157:H7 and S. Typhimurium, each diluted to $\sim 8.0 \log _{10} \mathrm{CFU} / \mathrm{mL}$. The inoculated samples were then submerged in solutions containing nanoparticles loaded with geraniol (GPN; 0.5 wt.\% geraniol), unencapsulated geraniol (UG; 0.5 wt.\%), or 200 ppm chlorine ( $\mathrm{HOCl}$; pH 7.0), with untreated samples serving for controls. Following antimicrobial treatment application, samples were collected for surviving pathogen enumeration or were placed under refrigeration $\left(5^{\circ} \mathrm{C}\right)$ for up to 10 days, with periodic enumeration of pathogen loads. After 3 days of refrigerated storage, all samples were removed, aseptically opened and subjected to a second inoculation with both pathogens. Treatment of spinach surfaces with encapsulated geraniol reduced both pathogens to non-detectable numbers within 7 days of refrigerated storage, even with a second contamination event occurring 3 days after experiment initiation. Similar results were observed with the UG treatment, except that upon recontamination at day 3, a higher pathogen load was detected on UG-treated spinach vs. GPN-treated spinach. These data fill a research gap by providing a novel tool to reduce enteric bacterial pathogens on spinach surfaces despite multiple contamination events, a potential food safety risk for minimally processed edible produce.

Keywords: antimicrobial nanoparticles, enteric pathogens, spinach, multiple contaminations, plant-derived antimicrobials, produce safety 


\section{INTRODUCTION}

Foodborne diseases caused by bacterial enteric pathogens, including Salmonella and Shiga toxin-producing Escherichia coli (STEC) are repeatedly linked to the consumption of crosscontaminated foods, resulting in hospitalizations and fatalities. These result in substantial losses in wages, quality of life, and other financial costs (Scallan et al., 2011; Batz et al., 2012). Fresh and minimally processed produce, including leafy vegetables such as lettuce and spinach, have been identified by public health specialists as significant food vehicles in the transmission of bacterial human pathogens. Painter et al. (2013) reported produce accounted for $46 \%$ of human foodborne illnesses occurring in the U.S, with leafy vegetables as the leading produce commodity type associated with pathogen transmission and human disease, followed by fruits and nuts. Over the last 5 years, the U.S. Centers for Disease Control and Prevention (CDC) reported five human disease outbreaks involving leafy green vegetables as vehicles transmitting Escherichia coli O157:H7, including two outbreaks in 2018 and one in 2019 involving romaine lettuce (CDC, 2018, 2019, 2020). Astill et al. (2020a,b) suggested reasons for these observed trends, including the high volume of leafy green consumption per capita in the U.S. leading to increased pathogen exposure risk, and seasonality- and climate-related factors influencing pre-harvest contamination in lettuce production fields and atmospheric temperatures supporting enteric pathogen survival on leaf surfaces. Similarly, between 1973 and 2011, researchers identified 19 U.S. outbreaks of foodborne disease involving cantaloupes, wherein Salmonella was the most commonly identified etiologic agent, causing 1,012 recorded disease cases and 38 fatalities, including one fetal death (Walsh et al., 2014). Burris et al. (2021) recently discussed Salmonella capabilities to contaminate melon fruit through blossom contamination as well as internalization into cantaloupe flesh as drivers of melon transmission of the pathogen to consumers.

Produce contamination with human pathogenic microbes may occur at multiple points along the production to plate continuum, and there are multiple opportunities for crosscontamination. Because produce is often consumed raw or after only minimal processing, a sanitizing treatment is often recommended to protect consumer safety (Fatica and Schneider, 2011; Castro-Ibáñez et al., 2017), though the most important barrier to pathogen transmission in fresh produce is contamination prevention. The U.S. Food and Drug Administration (FDA) final rule for the protection of safety of fresh produce requires produce growers subject to the rule to take steps to protect produce safety from identified food safety hazards, including microbial pathogens (2020). This can include the treatment of waters contacting harvested produce with a sanitizer to prevent contamination between produce items.

Plant-derived antimicrobials (PDAs) make attractive produce disinfectants due to their natural origin, compatibility with various produce commodities, and utility against microbial pathogens (Yoon and Lee, 2018). Nonetheless, PDA utility is often limited due to inherent volatility and, in some instances, low water solubility (Ruengvisesh et al., 2019b). One strategy to overcome these limitations of PDAs is to encapsulate them in a water-miscible system to facilitate their delivery to produce surfaces, potentially improving on observed antimicrobial effects (Yegin et al., 2016). The application of nanoparticles constructed of the tri-block co-polymer Pluronic F-127 loaded with the PDA geraniol (trans-3,7-Dimethyl-2,6-octadien-1-ol) inhibited the growth of E. coli $\mathrm{O} 157: \mathrm{H} 7$ and S. Typhimurium on spinach over 10 days of refrigerated storage, reducing pathogen numbers to non-detection within 5 days of experiment initiation (Perez-Lewis et al., 2018). Geraniol has been reported to exert antimicrobial activity against bacterial organisms through its impacts on membrane components, resulting from significant destabilization of membrane phospholipid arrangement and packing, as well as through formation of aggregates of lipids leading to increase leakage of solutes (Nowotarska et al., 2014). These antimicrobial-bearing nanoparticles also reduced pathogen loads on spinach surfaces to $<1.0 \log _{10} \mathrm{CFU} / \mathrm{cm}^{2}$ when spinach was inoculated after applying antimicrobial treatment, an outcome not observed for unencapsulated PDA or $200 \mathrm{ppm}$ hypochlorous acid treatments.

Despite the wealth of information and research data available, studies seeking to determine the sanitizing potential of produce sanitizers under conditions simulating multiple contamination events (i.e., pre-harvest and post-harvest) are missing from the literature, though the opportunity for produce to be repeatedly contaminated during its production and post-harvest handling is plausible. Therefore, the primary objective of this research was to determine the capability of treatment with encapsulated or free geraniol, or free chlorine $(200 \mathrm{mg} / \mathrm{L})$, to decontaminate spinach leaves during refrigerated storage when antimicrobial treatment was applied after a simulated pre-harvest contamination but before post-harvest simulated cross-contamination.

\section{MATERIALS AND METHODS}

\section{Preparation of Geraniol-Loaded Nanoparticles and Nanoparticle Characterization}

Geraniol (CAS \#1-6-24-1; TCI America, Portland, OR, USA) and Pluronic F-127 (CAS \#9003-1-6; Sigma-Aldrich Corp., St. Louis, MO, USA) were dissolved at a 1:1 ratio in tetrahydrofuran (CAS \#109-99-9; Sigma-Aldrich Corp.; THF), each to 8.0 wt.\% for $10 \mathrm{~min}$ using a probe ultra-sonicator (SJIA-2000W; Ningbo Haishu Sklon Electronics Instruments Co., Ltd., Ningbo, Zhejiang, China). Pluronic F-127, also known as poloxamer 407 , is currently allowed by the U.S. FDA for use in various pharmaceutical manufacturing processes, including some ingested orally, to a maximum content of $117 \mathrm{mg}$ per dosage, to provide entrapment and extended release capabilities to prescribed drugs (FDA, 2021). Following mixing of THFdissolved geraniol and Pluronic F-127, the mix was slowly added in milli-Q water, to a ratio of 1:10 (emulsion mix:sterile milli$\mathrm{Q}$ water) and sonicated for $15 \mathrm{~min}$ at $25 \pm 1^{\circ} \mathrm{C}$ (Figure 1). Crude GPNs $(20.0 \mathrm{~mL})$ were added into standard regenerated cellulose membranes (molecular weight cut-off 12-14 kDa; $\sim 2.0 \mathrm{~nm}$ diameter cut-off) (Spectrum Laboratories, Inc., Rancho 

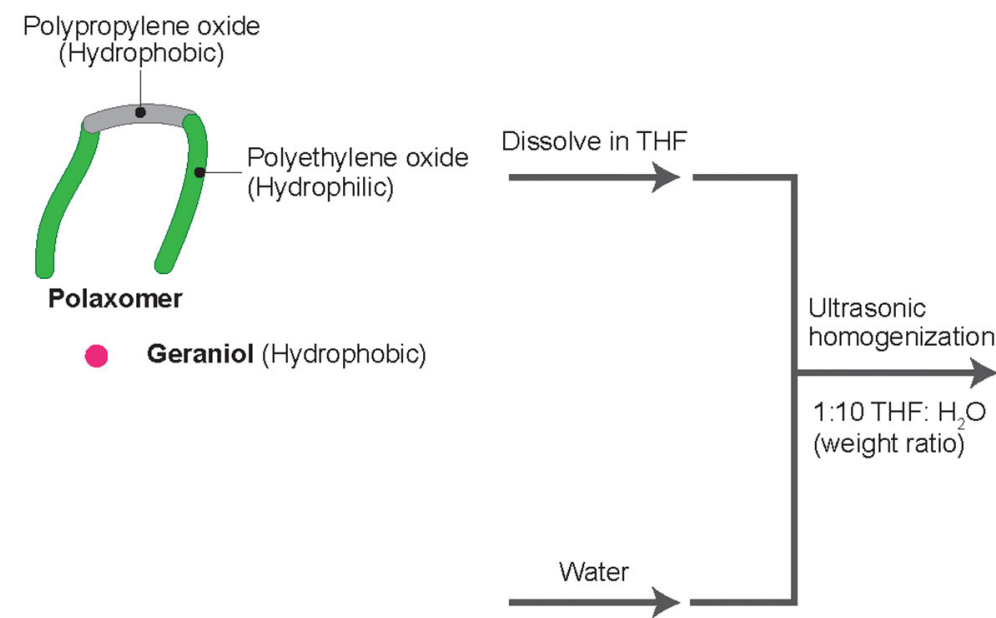

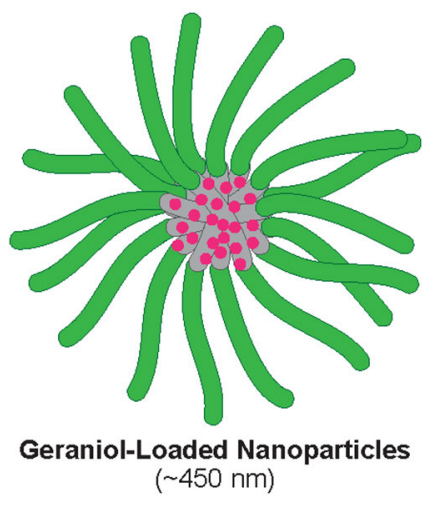

FIGURE 1 | Preparation of geraniol-loaded polymeric nanoparticles (GPNs).

Dominguez, CA), then the membrane was placed into beakers containing 2.0 L milli-Q water for an overnight dialyses $(12.0 \mathrm{~h}$; $25 \pm 1^{\circ} \mathrm{C}$ ) to remove THF and unencapsulated geraniol. THF and unencapsulated geraniol were passively diffused through dialysis membrane while encapsulated geraniol stayed in the dialysis membrane. The final concentration of encapsulated geraniol was 0.5 wt. $\%$ in milli-Q water, with an $\sim 57.5 \pm 5.5 \%$ encapsulation efficiency determined by mass spectrometry (MS), as previously reported (Yegin et al., 2016).

GPNs were subjected to particle size characterization by dynamic light scattering (DLS) following a 4-fold dilution in milli-Q water in a Zetasizer ZS90 analyzer (Malvern Instruments, Ltd., Westborough, MA, USA). Measurements were carried out at a $90^{\circ}$ scattering angle, at $25^{\circ} \mathrm{C}$ to determine GPN sizes and homogeneity. Subsequently, GPN morphology and sizes were evaluated by scanning electron microscopy (SEM) using a JSM7500F electron microscope (JEOL, Tokyo, Japan). Before coating, one drop of GPNs was dropped onto a silicon wafer and dried under a fume hood. Prior to imaging, samples were coated with $3 \mathrm{~nm}$ of Platinum/Palladium (Pt/Pd) with a sputter coater to ensure electrical conductivity. The electron microscope was operated at an emission current of $20.0 \mu \mathrm{A}$ and accelerating voltage of $5.0 \mathrm{kV}$.

\section{Storage Stability of Geraniol-Loaded Nanoparticles to Temperature- and pH-Abuse}

In order to determine the stability of GPNs to exposure to conditions of storage abuse, GPNs were prepared as described in section Preparation of Geraniol-Loaded Nanoparticles and Nanoparticle Characterization and thereafter stored at differing temperature $\left(4,25,37\right.$, and $\left.50^{\circ} \mathrm{C}\right)$ at $\mathrm{pH} 7.0$. In a separate experiment, GPNs were prepared and loaded into reaction vessels pre-conditioned to $\mathrm{pH} 4.0$, or 10.0 at ambient temperature; storage solutions were diluted and $\mathrm{pH}$-adjusted to desired $\mathrm{pH}$ with either $\mathrm{HCl}$ or $\mathrm{NaOH}(0.133 \mathrm{mM}$ ea.). In both experiments,
GPNs subjected to temperature- or $\mathrm{pH}$-abuse conditions were sampled at $0,1,2,4,7,14,30$, and 60 days of storage and subjected to DLS analysis as described above (section Preparation of Geraniol-Loaded Nanoparticles and Nanoparticle Characterization). Hydrochloric acid ( $\mathrm{HCl} ; 36.5-38.0 \%)$ (VWR, Inc.), and $\mathrm{NaOH}$ (>98.0\%; Sigma-Aldrich Co.) were purchased and used as received.

\section{Preparation of Microorganisms for Inoculation Onto Spinach Sample Surfaces}

Isolates of $S$. Typhimurium LT2 (American Type Culture Collection, Manassas, VA, USA [ATCC] \#700720) and stxnegative E. coli O157:H7 ATCC \#700728, resistant to the antibiotic rifampicin at $100.0 \mu \mathrm{g} / \mathrm{mL}$, were obtained from Food Microbiology Laboratory (Department of Animal Science, Texas A\&M AgriLife, College Station, TX, USA) culture collection and prepared by first aseptically passing into tryptic soy broth (Becton, Dickinson and Co., Sparks, MD, USA) followed by $24 \mathrm{~h}$ incubation at $35^{\circ} \mathrm{C}$. A second passage for each organism was completed in identical fashion to revive and prepare cells for subsequent inoculum preparation. Organisms were selected to accommodate biosafety needs for already published research conducted simultaneous to data collection in the current study (Yegin et al., 2016). Previous research has indicated these and other similar virulence-attenuated organisms functioned effectively as pathogen surrogates for evaluating decontamination of stone fruit (Yun et al., 2013), or have demonstrated utility for discerning pathogen spread during leafy vegetable packing in research pilot facilities (Smolinski et al., 2018).

An inoculum of the microorganisms was prepared by first centrifuging microbial isolates for $15 \mathrm{~min}$. at $2,191 \times g$ at ambient temperature to pelletize cells. Resulting supernatants were carefully poured off and discarded, and bacterial pellets were mixed in $10.0 \mathrm{~mL}$ of sterile phosphate-buffered saline (Thermo-Fisher Scientific, Waltham, MA, USA) to wash cells. 
Centrifugation and washing of cell pellets was completed three times in identical fashion before cells were blended together and then serially diluted to a target of $8.0 \log _{10}$ $\mathrm{CFU} / \mathrm{mL}$ in $0.1 \%$ peptone diluent (Becton, Dickinson and Co.). Numbers of E. coli O157:H7 and S. Typhimurium in inoculum fluid were differentially counted by preparation of serial dilutions in $0.1 \%$ peptone diluent and plating on surfaces of lactose-sulfite-phenol red-rifampicin (LSPR) agar supplemented with $100.0 \mu \mathrm{g} / \mathrm{mL}$ rifampicin. Reagents to produce LSPR were purchased individually from Becton, Dickinson and Co. and Sigma-Aldrich Co. (St. Louis, MO, USA) and were of highest quality. Inoculated Petri dishes were incubated for $24-36 \mathrm{~h}$ at $36 \pm 1{ }^{\circ} \mathrm{C}$ prior to colony counting. E. coli O157:H7 creamcolored colonies displayed a halo of lactose fermentation, while Salmonella colonies displayed black centers due to sulfite precipitation, with no haloes of fermented lactose (Castillo et al., 1998; Perez-Lewis, 2015).

\section{Inoculation of Spinach Samples Simulating Pre-harvest Contamination}

Spinach was purchased from a College Station, TX produce wholesaler and returned to the Food Microbiology Laboratory upon pickup. Spinach leaves were rinsed in sterile distilled water and allowed to drip dry on sterile stainless steel racks for $1.0 \mathrm{~h}$ prior to portioning into $10 \mathrm{~cm}^{2}$ circular pieces by use of a flamesterilized $10 \mathrm{~cm}^{2}$ disc borer to function as samples. Samples were composed of three pieces $\left(10 \mathrm{~cm}^{2}\right.$ per piece), giving each sample a total of $30 \mathrm{~cm}^{2}$ inoculated and treated surface area. Samples were spot-inoculated with ten $10.0 \mu \mathrm{L}$ volumes containing pathogens onto adaxial surfaces of leaves (abaxial surfaces were placed face down and not inoculated). Samples were then held at $25^{\circ} \mathrm{C}$ for $1.0 \mathrm{~h}$ prior to sanitizing treatment to allow inoculated cells to attach to leaf surfaces.

\section{Antimicrobial Treatment Application and Inoculation of Samples Simulating Post-harvest Contamination}

Sanitizing treatments were applied to inoculated samples by submerging samples in sanitizer-containing solutions $(20.0 \mathrm{~mL}$ per sample) for two min at ambient temperature $\left(25 \pm 1^{\circ} \mathrm{C}\right)$ in sanitizer-treated waters. Treatments were geraniol nanoparticles (GPNs) containing 0.5 wt.\% geraniol, unencapsulated geraniol (UG; 0.5 wt.\%) diluted in sterile milli-Q water, $200 \mathrm{ppm}$ free/available chlorine titrated to $\mathrm{pH} 7.0(\mathrm{HOCl})$, and a no sanitizer-containing sterile water control. The $\mathrm{HOCl}$ treatment was prepared by dilution of $6.25 \% \mathrm{NaOCl}$ bleach sanitizer (Clorox Co., Oakland, CA, USA) in sterile distilled water prior to $\mathrm{pH}$ adjustment with $1.0 \mathrm{~N} \mathrm{HCl}$. The $\mathrm{HOCl}$ treatment was checked for chlorine concentration by free/total chlorine test strip (Hach Co., Loveland, CO, USA). Following the $2 \mathrm{~min}$ of sanitizing treatment application, sample pieces were aseptically removed from treatment with sterile forceps, placed for $15 \mathrm{~min}$ on a sanitary paper towel to drip dry, and then assigned to either immediate microbiological assay or to a storage period (3, 5, 7 , or 10 days). Samples were not rinsed or otherwise treated to remove sanitizing treatment residue prior to microbiological analysis or refrigerated storage. Samples assigned to a storage period were then placed in sterile plastic Petri dishes $(100 \times$ $15 \mathrm{~mm}$; volume $\left.=1.8 \times 10^{5} \mathrm{~mm}^{3}\right)$, covered with an oxygentransmitting film (low density polyethylene), and stored in a refrigerated incubator set to hold temperature at $5 \pm 1{ }^{\circ} \mathrm{C}$. Samples were only subjected to a sanitizing treatment once, following the initial pathogen inoculation event; no subsequent sanitizing treatments were performed, in order to determine the ability of differing sanitizing treatments to exhibit pathogen growth inhibition following treatment and a second pathogen inoculation (simulated cross-contamination) occurrence.

To determine the ability of sanitizing treatments to inhibit pathogen growth on samples subjected to two sequential pathogen inoculation events, stored samples were removed from refrigeration after 3 days of storage and inoculated in like fashion as described in section Inoculation of Spinach Samples Simulating Pre-harvest Contamination. For the second inoculation event, inoculum fluid (containing a blend of both bacterial organisms) was adjusted to $\sim 7.0 \log _{10}$ $\mathrm{CFU} / \mathrm{mL}$ according to the suggestion that post-harvest crosscontamination does not likely occur at numbers higher than those expected to occur in a pre-harvest contamination event (Harris et al., 2003). For microbiological analysis of organisms on spinach sample surfaces, all samples were either subjected to selective/differential enumeration of inoculated microbes on LSPR agar following preparation of serial dilutions in $0.1 \%$ peptone diluent and subsequent incubation for $24-36 \mathrm{~h}$ at 36 $\pm 1{ }^{\circ} \mathrm{C}$ (section Preparation of Microorganisms for Inoculation Onto Spinach Sample Surfaces), or were returned to refrigerated storage prior to later pathogen enumeration. Serial dilutions were initiated by mixing of spinach samples in $100 \mathrm{~mL}$ sterile $0.1 \%$ peptone diluent and stomaching at $230 \mathrm{rpm}$ for $1.0 \mathrm{~min}$ prior to preparation of subsequent 10 -fold dilutions.

\section{Experimental Design and Data Analysis}

Characterization experiment of GPNs size by DLS and environmental stability was carried out using a factorial arrangement and replicated twice, with duplicate analytical samples taken from a larger GPN preparation $(N=2)$. The antimicrobial activity experiment was designed as a factorial arrangement of a completely randomized design, replicated three times in identical fashion $(N=3)$. Samples not selected for immediate enumeration on an experiment day 0 were randomly assigned to a storage period (3, 5, 7, or 10 days) before storing at $5^{\circ} \mathrm{C}$, yielding a total of 60 distinct spinach samples for use in antimicrobial treatment experimentation. All microbiological data were transformed to $\log _{10} \mathrm{CFU} / \mathrm{cm}^{2}$ prior to data analysis. Data were analyzed by analysis of variance (ANOVA), evaluating the impacts of the main effects of antimicrobial treatment and storage period, as well as their interaction, on resulting survivor counts of pathogens. Pathogens were analyzed separately from one another. Statistical significance was assigned at $P<0.05$, and differing means of survivor counts were separated by Tukey's Honestly Significant Differences (HSD) post-hoc test. Data were analyzed using JMP ${ }^{\circledR}$ Pro v15.0 for Macintosh (SAS Institute, Inc., Cary, NC, USA). 


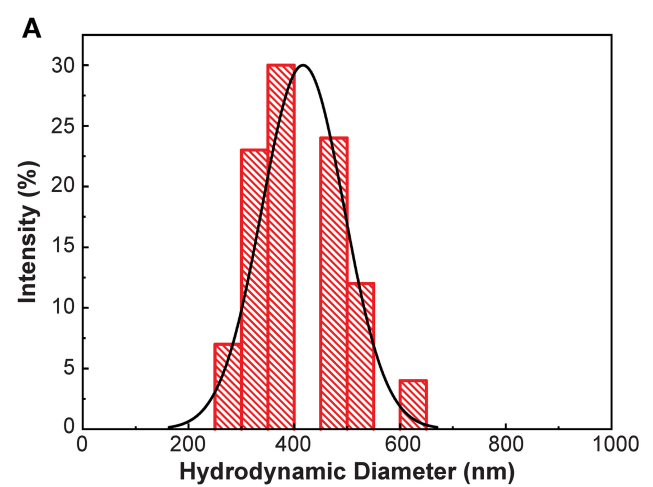

B

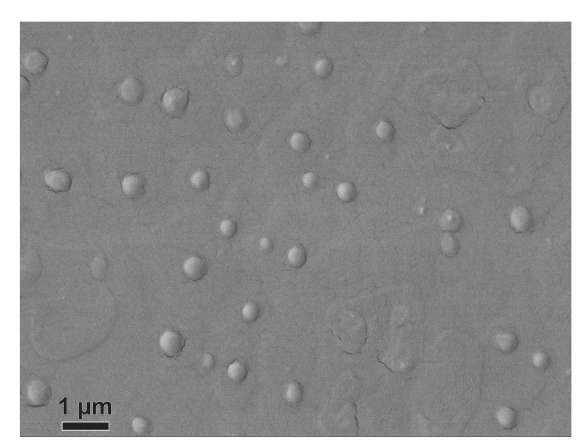

FIGURE 2 | Particle size distribution (A) and scanning electron microscopy image (B) of geraniol-loaded nanoparticles.

\section{RESULTS}

The current study sought to evaluate the produce surfacesanitizing utility of an innovative antimicrobial treatment composed of polymeric nanoparticles loaded with the PDA geraniol, as well as free geraniol and $200 \mathrm{ppm}$ free chlorine, in a scenario involving multiple sequential spinach contamination events. In previous research, identically-prepared GPNs prevented growth of these organisms on spinach surfaces during refrigerated and temperature abuse storage, and showed capacity to inhibit pathogen growth when applied prior to pathogen inoculation (Perez-Lewis et al., 2018). Figure 2A depicts the size distribution of GPNs via DLS analysis, and indicates a mean particle size of $416.5 \pm 77.9 \mathrm{~nm}$. Electron microscopy analysis confirmed DLS findings with respect to particle size and size distribution (Figure 2B).

Changes in hydrodynamic sizes of GPNs measured by DLS over 60 days as function of temperature and $\mathrm{pH}$ abuse are shown in Figures 3, 4, respectively. At $25^{\circ} \mathrm{C}$, particle size did not change as a function of storage period, demonstrating good storage stability at ambient temperature $(P>0.05)$. Conversely, mean diameters of GPNs stored at $4^{\circ} \mathrm{C}$ differed from those of GPNs held at $25^{\circ} \mathrm{C}$ up to day 14 (Figure 3). Conversely, at $37^{\circ} \mathrm{C}$, although the size of GPNs did not change during storage for the majority of particles, a second population of particles with a mean diameter of $\sim 37.36 \pm 25.57 \mathrm{~nm}$ was detected at day 14 of storage. This second population of GPNs was also detected at 30 days of storage, but by 60 days the diameter of both populations of GPNs were nearly identical (Figure 3). Samples taken from GPNs stored at $50^{\circ} \mathrm{C}$ also revealed a second population of smaller GPNs that initially decreased in diameter through 14 days of storage without further decrease in diameter thereafter through the end of the experimental period (Figure 3). The GPNs stored at 50 ${ }^{\circ} \mathrm{C}$ showed a second peak detected at the 2 nd day of storage. However, unlike GPNs held at $37^{\circ} \mathrm{C}$, the second population of GPNs from $50^{\circ} \mathrm{C}$ incubation did not increase in size during storage, rather remaining constant up to day 14. Surprisingly, a second population of GPNs with smaller diameter was also determined to exist in NP solution stored at $4^{\circ} \mathrm{C}$. As with samples stored at $37^{\circ} \mathrm{C}$, the mean diameter of this second population

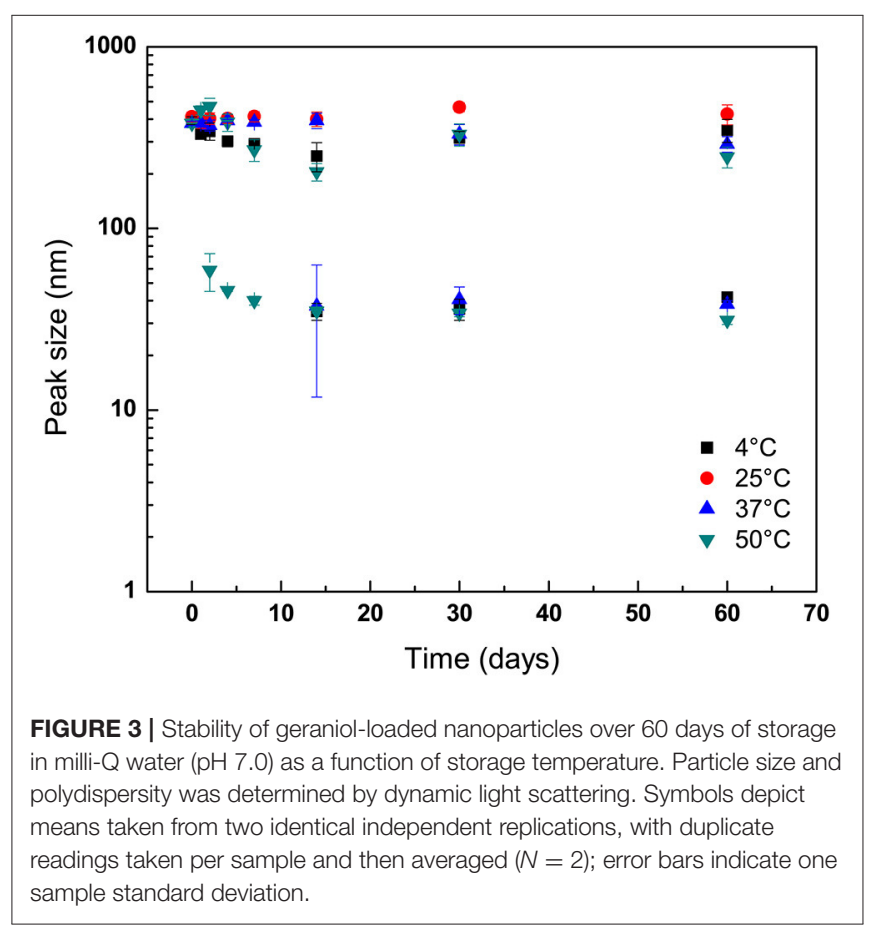

of GPNs was initially observed at 14 days of storage, though it behaved more like GPNs stored at $50^{\circ} \mathrm{C}$ in that its mean diameter did not increase over storage but rather decreased until a point at which it remained static thereafter.

Nanoparticles were loaded into solutions adjusted to $\mathrm{pH} 4.0$, 7.0, or 10.0, and then stored at $25^{\circ} \mathrm{C}$ (Figure 4). No differences in GPN mean diameters were observed for samples taken from the three storage conditions through the first 14 days (Figure 4). However, evidence of GPN degradation was detected at day 30 for $\mathrm{pH} 4.0$-stored samples as compared to those held under neutral and alkaline $\mathrm{pH}$ conditions, with the detection of a second population of smaller GPNs with a mean diameter of $\sim 79.05 \pm$ $35.86 \mathrm{~nm}(P<0.05)$. Interestingly, in GPNs stored at $\mathrm{pH} 10.0$, 


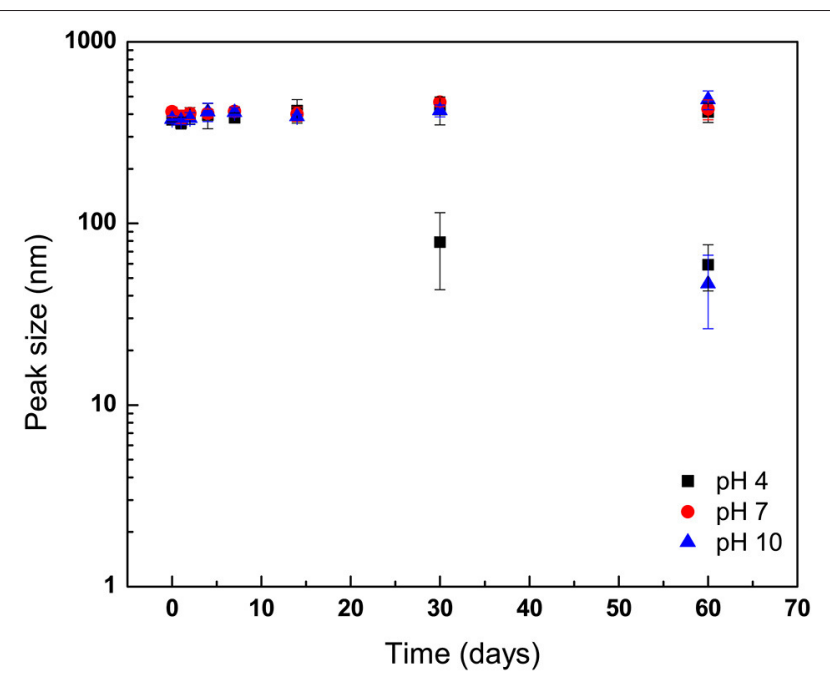

FIGURE 4 | Stability of geraniol-loaded nanoparticles over 60 days of storage in milli- $\mathrm{Q}$ water $\left(25^{\circ} \mathrm{C}\right)$ as a function of storage $\mathrm{pH}$. Storage solution $\mathrm{pH}$ was modified by controlled addition of $0.133 \mathrm{mM} \mathrm{HCl}$ or $\mathrm{NaOH}$. Particle size and polydispersity was determined by dynamic light scattering. Symbols depict means taken from two identical independent replications, with duplicate readings taken per sample and then averaged $(N=2)$; error bars indicate one sample standard deviation.

evidence of development of a bimodal distribution of GPNs was not observed until day 60 of the incubation period.

Statistical analysis of microbiological data resulted in the identification of a significant interaction of the main effects of antimicrobial treatment by storage period with respect to counts of surviving E. coli O157:H7 and S. Typhimurium. The GPN treatment produced significantly greater reductions in pathogens vs. the $\mathrm{HOCl}$ and Control treatments, though GPNs did not outperform the UG treatment with respect to the numbers of surviving pathogens at 7 and 10 days of refrigerated storage. The mean count of inoculated E. coli O157:H7 following the first inoculation event, simulating pre-harvest contamination, was $5.6 \pm 0.6 \log _{10} \mathrm{CFU} / \mathrm{cm}^{2}$. By comparison, E. coli $\mathrm{O} 157: \mathrm{H} 7$ attaching to spinach surfaces following the second inoculation event averaged $5.5 \pm 0.3 \log _{10} \mathrm{CFU} / \mathrm{cm}^{2}$. Immediately following inoculation and antimicrobial treatment (day 0), all treatments except the Control reduced E. coli O157:H7 on spinach, with geraniol treatments $(\mathrm{GPN}, \mathrm{UG})$ reducing the pathogen to the limit of detection $\left(0.5 \log _{10} \mathrm{CFU} / \mathrm{cm}^{2}\right)$. HOCl reduced the pathogen by only $2.3 \log _{10}$-cycles, significantly less than the reductions achieved by the geraniol-using treatments $(P<0.05)$. Numbers of the pathogen rose again on day 3 following the second inoculation event, though numbers of E. coli $\mathrm{O} 157: \mathrm{H} 7$ on GPN-treated spinach rose only modestly from day 0 , and were not different from pathogen numbers on any other day for GPN-treated samples. In contrast, numbers of E. coli O157:H7 on UG- and HOCl-treated spinach rose sharply after the second inoculation to 4.3 and $5.0 \log _{10} \mathrm{CFU} / \mathrm{cm}^{2}$, respectively, though UG treatment produced a greater decline in E. coli O157:H7 than did $\mathrm{HOCl}$ treatment following the second contamination
TABLE 1 | Least squares means of surviving Escherichia coli O157:H7 $\left(\log _{10}\right.$ $\mathrm{CFU} / \mathrm{cm}^{2}$ ) on spinach leaf surfaces by the interaction of antimicrobial treatment $\mathrm{x}$ storage period.

\begin{tabular}{lccccc}
\hline & \multicolumn{5}{c}{ Storage period (days) $^{\mathbf{b}}$} \\
\cline { 2 - 6 } Treatment $^{\mathbf{a}}$ & $\mathbf{0}$ & $\mathbf{3}^{\mathbf{c}}$ & $\mathbf{5}$ & $\mathbf{7}$ & $\mathbf{1 0}$ \\
\hline $\mathrm{GPN}$ & $0.5 \mathrm{E}$ & $1.7 \mathrm{DE}$ & $0.5 \mathrm{E}$ & $0.5 \mathrm{E}$ & $0.5 \mathrm{E}$ \\
UG & $0.5 \mathrm{E}$ & $4.3 \mathrm{AB}$ & $2.3 \mathrm{CDE}$ & $0.5 \mathrm{E}$ & $0.5 \mathrm{E}$ \\
$\mathrm{HOCl}$ & $3.1 \mathrm{BCD}$ & $5.0 \mathrm{AB}$ & $4.4 \mathrm{AB}$ & $3.7 \mathrm{ABC}$ & $3.1 \mathrm{BCD}$ \\
Control & $5.6 \mathrm{~A}$ & $5.5 \mathrm{~A}$ & $5.6 \mathrm{~A}$ & $4.6 \mathrm{AB}$ & $3.4 \mathrm{BCD}$ \\
$P=0.0004 ;$ & Pooled SEM & & & &
\end{tabular}

a Treatments are GPN (nanoparticles loaded with geraniol to 0.5 wt.\%), UG (free geraniol at 0.5 wt.\%), HOCl (200.0 ppm free chlorine at $\mathrm{pH}$ 7.0), and Control (untreated). Treatments were applied by immersion for 2.0 min prior to samples being removed from antimicrobial treatment, placed in sterile covered containers, and stored at $5^{\circ} \mathrm{C}$. Mean numbers of $\mathrm{E}$. coli 0157:H7 cells inoculated onto samples initially were $5.6 \pm 0.6 \log _{10} \mathrm{CFU} / \mathrm{cm}^{2}$ and were $5.5 \pm 0.3 \log _{10} \mathrm{CFU} / \mathrm{cm}^{2}$ after the second inoculation at storage day 3 .

${ }^{b}$ Values depict means of three identically completed replicates $(N=3)$; means not sharing letters $(A, B, C, \ldots)$ differ at $P=0.05$ by Tukey's Honestly Significant Differences (HSD) test. cSpinach samples were re-inoculated with E. coli 0157:H7 (7.0 $\log _{10}$ CFU/mL); all samples assigned to storage periods of 3, 5, 7, and 10 were re-inoculated in identical fashion.

dSEM, Standard error about the mean.

event. The Control yielded only a very modest reduction in pathogen numbers over the 10-day storage period, potentially the result of some minimal washing of loosely adhered cells at experiment's start and subsequent low temperature storage impacts on microbial survival (Table 1).

Similar differences were observed in the evolution of counts of surviving S. Typhimurium cells following the initial inoculation event, antimicrobial treatment application, and refrigerated storage with re-inoculation after 3 days of storage (Table 2). The mean count of inoculated $S$. Typhimurium cells following the first inoculation event was $5.9 \pm 0.6 \log _{10} \mathrm{CFU} / \mathrm{cm}^{2}$. By comparison, counts of cells attaching to spinach surfaces following the second inoculation event averaged $5.6 \pm 0.5 \log _{10}$ $\mathrm{CFU} / \mathrm{cm}^{2}$. For both E. coli O157:H7 and S. Typhimurium, counts were rapidly reduced to the limit of detection (day 0 ) by the GPN treatment. Pathogen numbers rose following the second inoculation (simulated re-contamination) event on day 3 , but declined again to the limit of detection by the 5 th day of refrigerated storage (Table 2). The UG treatment reduced the pathogen to the limit of detection after 10 days of refrigerated storage, as compared to 7 days in the case of E. coli O157:H7 (Table 1). Chlorine (i.e., $\mathrm{HOCl}$ ) treatment produced a reduction in pathogen numbers over refrigerated storage, but not to the extent that geraniol-applying treatments (GPN, UG) produced. Finally, the Control yielded only a slow decline in Salmonella numbers, again the likely result of the prolonged cold storage conditions preventing Salmonella replication.

\section{DISCUSSION}

The current study presents the findings of experiments designed to test whether a produce food safety intervention applied onto a model produce commodity (spinach leaves) would be 
TABLE 2 | Least squares means of surviving Salmonella Typhimurium ( $\log _{10}$ $\mathrm{CFU} / \mathrm{cm}^{2}$ ) on spinach leaf surfaces by the interaction of antimicrobial treatment $\mathrm{x}$ storage period.

\begin{tabular}{|c|c|c|c|c|c|}
\hline \multirow[b]{2}{*}{ Treatment $^{a}$} & \multicolumn{5}{|c|}{ Storage period (days) ${ }^{b}$} \\
\hline & 0 & $3^{c}$ & 5 & 7 & 10 \\
\hline GPN & $0.5 \mathrm{G}$ & 1.5EFG & 1.0FG & $0.5 \mathrm{G}$ & $0.5 \mathrm{G}$ \\
\hline UG & 1.3FG & 4.2ABCD & 2.5DEFG & $0.6 \mathrm{G}$ & $0.5 \mathrm{G}$ \\
\hline $\mathrm{HOCl}$ & 4.0ABCD & $5.1 \mathrm{AB}$ & 4.4ABCD & 3.5BCDE & 2.8CDEF \\
\hline Control & $5.9 \mathrm{~A}$ & 5.6AB & 5.7AB & 4.7ABC & 3.5BCDE \\
\hline
\end{tabular}

a Treatments are GPN (nanoparticles loaded with geraniol to 0.5 wt.\%), UG (free geraniol at 0.5 wt.\%), $\mathrm{HOCl}$ (200.0 ppm free chlorine at pH 7.0), and Control (untreated). Treatments were applied by immersion for $2.0 \mathrm{~min}$ prior to samples being removed from antimicrobial treatment, placed in sterile covered containers, and stored at $5^{\circ} \mathrm{C}$. Mean numbers of $\mathrm{S}$. Typhimurium cells inoculated onto samples initially were $5.9 \pm 0.6 \log _{10} \mathrm{CFU} / \mathrm{cm}^{2}$ and were $5.6 \pm 0.3 \log _{10} \mathrm{CFU} / \mathrm{cm}^{2}$ after the second inoculation at storage day 3 .

${ }^{b}$ Values depict means of three identically completed replicates $(N=3)$; means not sharing letters $(A, B, C, \ldots)$ differ at $P=0.05$ by Tukey's Honestly Significant Differences (HSD) test. 'Spinach samples were re-inoculated with S. Typhimurium (7.0 $\left.\log _{10} \mathrm{CFU} / \mathrm{mL}\right)$; all samples assigned to storage periods of 3, 5, 7, and 10 were re-inoculated in identical fashion.

dSEM, Standard error about the mean.

effective at preventing the growth of enteric bacterial pathogens under conditions of simulated pre-harvest and post-harvest contamination events, data which are absent from the scientific literature to the authors' awareness. Although authors are unaware of a foodborne disease outbreak involving fresh spinach or other leafy greens wherein multiple pathogen contamination events were reported to occur, it is not unreasonable to suggest that produce commodities are not subject to such insanitary handling.

The development of a second population of nanoparticles held at $37^{\circ} \mathrm{C}$ may be explained as GPNs that released geraniol payload early in the storage period, with subsequent aggregation of polymer into complexes of polymer molecules with larger hydrodynamic radii than that for single polymer molecules over the course of storage. This observation, along with findings on evolution of nanoparticle size for those held at $50^{\circ} \mathrm{C}$ are not surprising and indicate the expulsion of geraniol early on with a likely reassembly of micellar structures. Prud'homme et al. (1996) reported micelles of Pluronic F-127 in aqueous solution were favored as incubation temperature was increased over $30^{\circ} \mathrm{C}$ due to disappearance of large domains as poly(oxyethylene) chains were increasingly dehydrated and repulsive interactions were decreased. The GPNs stored at $50^{\circ} \mathrm{C}$ showed a second peak detected at the 2 nd day of storage. However, unlike GPNs held at $37^{\circ} \mathrm{C}$, the second population of GPNs from $50^{\circ} \mathrm{C}$ incubation did not increase in size during storage but were constant up to day 14. It has been observed previously that prolonged exposure to elevated temperature conditions can assist the release of entrapped drug, resulting in nanoparticle degradation (Hill and Gomes, 2014). As discussed previously, a second population of GPNs with smaller diameter was, to author's surprise, determined to exist in NP solution stored at $4^{\circ} \mathrm{C}$. This may have been the result of reduced hydrophobicity of the poly(oxypropylenes) at the reduced storage temperature, which may have led to loss of emulsification of the hydrophobic oil and breakdown of the micelles, as well as the potential for unimers of Pluronic F127 molecules to exist at temperatures below the micellization temperature at the concentration used in the present study (8.0 wt.\%; $\sim 24^{\circ} \mathrm{C}$ ) (Wanka et al., 1994; Pham Trong et al., 2008). The release of geraniol from GPNs held at $\mathrm{pH} 4.0$ was more rapid than what was observed for GPNs loaded into neutral $\mathrm{pH}$ conditions, a response to environmental storage conditions previously reported for drug- and antimicrobialdelivering nanoparticles (Gaysinsky et al., 2005, 2008; Wuang et al., 2011). Interestingly, in GPNs exposed to aqueous solution adjusted to $\mathrm{pH} 10.0$, the late onset of a second population of nanoparticles (after 60 days' storage) indicates that Pluronic F127 nano-particles are not long-term stable to acid hydrolysis but bear moderate stability to hydroxide attack.

Experimental results demonstrate that sanitizing treatments involving the PDA geraniol, free or encapsulated, outperformed a chlorine sanitizer treatment as well as a water rinse step (control). Plant-derived antimicrobials and various essential oil components have been previously studied and reported for their utility in fresh produce decontamination during extended storage periods by multiple research groups (Ayala-Zavala et al., 2009; Azizkhani et al., 2013; Kang and Song, 2018). Yossa et al. (2012) tested the interaction of antimicrobial treatment and storage period (up to 14 days at $4^{\circ} \mathrm{C}$ ) during experiments tracking E. coli O157:H7 and Salmonella enterica serovars on baby spinach leaves treated with $800 \mathrm{ppm}$ cinnamic aldehyde (with or without the use of $0.5 \%$ tween 20 to enhance delivery of the PDA) or 5 ppm free chlorine. Similar to the results presented in the current study, an interaction of these treatment effects (sanitizer use, storage period) was detected from statistical analysis of microbiological data. This group also published decontamination studies on romaine lettuce leaves cut to a uniform size $\left(6.0 \mathrm{~cm}^{2}\right)$ where $E$. coli $\mathrm{O} 157: \mathrm{H} 7$ or Salmonella inocula were treated with $5.0 \mathrm{ppm}$ chlorine or 800 ppm cinnamaldehyde emulsified in $0.5 \%$ Tween 20 (Yossa et al., 2013). In that study, researchers demonstrated greater reductions in both pathogens by chlorine treatment vs. emulsified essential oil, indicating that physical damage to the leaf did not negate antimicrobial outcomes from treatment. Zhu et al. (2020) reported interaction of antimicrobial treatment by storage period for edible films incorporating differing PDAs on leafy greens. For mature spinach, researchers reported a $3 \%$ carvacrol-incorporating apple-derived film reduced E. coli O157:H7 to below the limit of detection over 3 days of refrigerated storage, though by 7 days of storage at $4{ }^{\circ} \mathrm{C}$ pathogen numbers had risen to low but detectable numbers. These results do not mirror those obtained in the current study, where GPN treatment suppressed both E. coli O157:H7 and S. Typhimurium to the limit of detection over 10 days of refrigerated storage (Tables 1, 2).

The inclusion of a second simulated cross-contamination event after 3 days of refrigerated storage on samples resulted in increases in numbers of both pathogens as compared to experiment outset for all treatments, excepting the Control, an expected outcome of the second inoculation event. Previous research studies have indicated the sanitizing effect of chlorine 
and various PDAs applied onto leafy green surfaces when the sanitizing treatment was applied after microbial organisms were inoculated onto the harvested crop (Beuchat et al., 2004; Yossa et al., 2012; Ruengvisesh et al., 2015). These research studies, nevertheless, failed to investigate the potential for sanitizing treatment to maintain any residual activity in the event of subsequent microbial contamination, the focus of the current study. GPN-treated samples sustained the lowest observed changes in pathogen numbers following the second pathogen inoculation, with the exception of the Control. That UG- and HOCl-treated spinach surfaces sustained greater increases in pathogen numbers following the second inoculation event was unsurprising given the potential for these sanitizing agents to be either volatilized or degraded by organic matter (Hill et al., 2013; Yoon and Lee, 2018). Nonetheless, observed declines in the numbers of both bacterial pathogens on spinach samples treated by $\mathrm{HOCl}$ by the end of the experimental storage period were unexpected. These may have occurred due to the initial reductions achieved by $\mathrm{HOCl}$ application in combination with extended refrigerated storage which effectively halted bacterial replication on the leafy green.

Inoculation of E. coli O157:H7 or Salmonella Typhimurium onto surfaces of spinach twice, simulating pre-harvest and postharvest contamination events, resulted in increased numbers of pathogens on commodities immediately following the second simulated cross-contamination event. Antimicrobial treatments, nonetheless, reduced pathogens on treated samples, with geraniol-using treatments (GPN, UG) being more effective for reducing pathogen numbers as compared to $\mathrm{HOCl}$ and Control treatments. While the potential for multiple crosscontamination events should be low for produce packed and held in a sanitary environment, research has demonstrated the opportunity for post-harvest contamination of pathogens to occur even when sanitizing treatments are applied (Pao et al., 2012; Singh et al., 2018; Smolinski et al., 2018). This occurrence is one the FDA's final rule on produce safety aims to prevent by the mandatory application of food safety hazard reduction strategies (FDA, 2020). No reports have been found detailing the utility of produce sanitizers when the produce item is contaminated multiple times (pre-harvest and/or post-harvest). Hence, the current study evaluated the utility of antimicrobial treatments under conditions simulating such events. Antimicrobial-loaded nanoparticles that release a produce sanitizer slowly are more effective for producing longerterm pathogen inhibition than other antimicrobial strategies that produce an initial burst effect and then dissipate either due to dilution, volatilization, or degradation with accumulated organic matter (Yegin et al., 2016; Huang and Nitin, 2019). These data can be applied by produce packers to select riskreducing strategies for controlling food safety hazards even for scenarios involving potential multiple cross-contamination of fresh produce.

The research data presented in the current manuscript were collected simultaneously with data already previously published by our group, wherein we identified multiple potential study limitations (Perez-Lewis et al., 2018). In addition to the potential study limits discussed in that report, there remain potential concerns over the financial costs of nano-particle formulation and preparation, and the demand for "clean" food ingredients used in food manufacturing that may limit produce industry adoption (Mora-Huertas et al., 2010; Blanco-Padilla et al., 2014). Nevertheless, the authors are unaware of any other scientific studies describing the outcomes of research where human foodborne microbial pathogens, or even indicator organisms, were applied onto a food crop surface, subjected to sanitizing treatment, and then subsequently re-inoculated in order to discern the capability of sanitizing treatment to protect against microbial proliferation prior to consumption. Though such insanitary handling conditions and practices would not likely be encountered in large food crop harvesting and handling facilities, the opportunity for pathogens to contaminate food crops repeatedly, including leafy greens, is logical given the exposure of crops in the field and opportunity for non-hygienic handling during post-harvest packing.

We are not surprised by the observed differences in spinach decontamination outcomes for the GPN and $\mathrm{HOCl}$ treatments, given our previous published research on antimicrobial-loaded nanoparticles applied to decontaminate spinach (Perez-Lewis et al., 2018; Ruengvisesh et al., 2019a). Finally, the current study details the capability of the plant-derived antimicrobial geraniol, free and encapsulated within polymeric micelles, to reduce the numbers of inoculated E. coli $\mathrm{O} 157: \mathrm{H} 7$ and $S$. Typhimurium on spinach leaves during a simulated multicontamination spinach production and post-harvest handling scenario. These data represent the first of their kind to the authors' knowledge regarding the opportunity presented by experimental antimicrobial treatments to protect harvested produce from proliferation of contaminating bacterial enteric pathogens. Nano-encapsulated PDAs, as well as free PDAs, represent useful candidates for sustained and safe technologies for fresh produce microbiological safety protection.

\section{DATA AVAILABILITY STATEMENT}

The datasets generated for this study can be found in online repositories. The names of the repository/repositories and accession number(s) can be found at: https://doi.org/10.18738/ T8/GDRSGW. Data not stored on repository will be made available upon reasonable request to the corresponding author.

\section{AUTHOR CONTRIBUTIONS}

YY and KP-L prepared experimental samples for physical analysis and microbiological experiments, respectively, collected experimental data, and assisted in preliminary data analysis. CK provided biostatistics analysis supervision and assistance. LC-Z and $\mathrm{AC}$ provided guidance on spinach handling and preparation, fresh produce decontamination experimental design, and assisted in final manuscript editing. SL and MA supervised antimicrobial nanoparticle fabrication and physical analysis. TT oversaw microbiological analysis experiments, prepared manuscript draft, final manuscript draft submission to journal, led competitive 
grant development, and submission providing financial support for research. All authors contributed to the article and approved the submitted version.

\section{FUNDING}

This work was supported by Agriculture and Food Research Initiative [Grant No. 2011-67107-30028] from the USDA National Institute for Food and Agriculture. YY and KP-L received graduate assistantship support from Texas A\&M AgriLife, Department of Food Science and Technology, College

\section{REFERENCES}

Astill, G., Todd, J. E., and Page, E. T. (2020a). Lettuce Help: USDA Data on Shipments of Romaine Lettuce Can Inform Foodborne Illness Outbreak Investigations and Public Health Advisories. Available online at: https:// www.ers.usda.gov/amber-waves/2020/february/lettuce-help-usda-data-onshipments-of-romaine-lettuce-can-inform-foodborne-illness-outbreakinvestigations-and-public-health-advisories/ (accessed November 6, 2020).

Astill, G. M., Kuchler, F., Todd, J. E., and Page, E. T. (2020b). Shiga toxinproducing Escherichia coli (STEC) O157:H7 and romaine lettuce: source labeling, prevention, and business. Am. J. Public Health 110, 322-328. doi: 10.2105/AJPH.2019.305476

Ayala-Zavala, J. F., González-Aguilar, G. A., and del Toro-Sánchez, L. (2009). Enhancing safety and aroma appeal of fresh-cut fruits and vegetables using the antimicrobial and aromatic power of essential oils. J. Food Sci. 74, R84-R91. doi: 10.1111/j.1750-3841.2009.01294.x

Azizkhani, M., Elizaquível, P., Sánchez, G., Selma, M. V., and Aznar, R. (2013). Comparative efficacy of Zataria multiflora Boiss., Origanum compactum and Eugenia caryophyllus essential oils against E. coli O157:H7, feline calicivirus and endogenous microbiota in commercial baby-leaf salads. Int. J. Food Microbiol. 166, 249-255. doi: 10.1016/j.ijfoodmicro.2013.07.020

Batz, M. B., Hoffmann, S., and Morris, J. J. G. (2012). Ranking the disease burden of 14 pathogens in food sources in the United States using attribution data from outbreak investigations and expert elicitation. J. Food Prot. 75, 1278-1291. doi: 10.4315/0362-028X.JFP-11-418

Beuchat, L. R., Pettigrew, C. A., Tremblay, M. E., Roselle, B. J., and Scouten, A. J. (2004). Lethality of chlorine, chlorine dioxide, and a commercial fruit and vegetable sanitizer to vegetative cells and spores of Bacillus cereus and spores of Bacillus thuringiensis. J. Food Prot. 67, 1702-1708. doi: 10.4315/0362-028X-67.8.1702

Blanco-Padilla, A., Soto, K. M., Iturriaga, M. H., and Mendoza, S. (2014). Food antimicrobials nanocarriers. Sci. World J. 2014:837215. doi: $10.1155 / 2014 / 837215$

Burris, K. P., Simmons, O. D., Webb, H. M., Moore, R. G., Jaykus, L.-A., Zheng, J., et al. (2021). Salmonella enterica colonization and fitness in pre-harvest cantaloupe production. Food Microbiol. 93:103612. doi: 10.1016/j.fm.2020.103612

Castillo, A., Lucia, L. M., Goodson, K. J., Savell, J. W., and Acuff, G. R. (1998). Use of hot water for beef carcass decontamination. J. Food Prot. 61, 19-25. doi: 10.4315/0362-028X-61.1.19

Castro-Ibáñez, I., Gil, M. I., and Allende, A. (2017). Ready-to-eat vegetables: current problems and potential solutions to reduce microbial risk in the production chain. Food Sci. Technol. 85, 284-292. doi: 10.1016/j.lwt.2016.11.073

CDC (2018). Multistate Outbreak of E. coli O157:H7 Infections Linked to Romaine Lettuce. Available online at: https://www.cdc.gov/ecoli/2018/o157h7-04-18/ index.html (accessed July 7, 2019).

CDC (2019). Outbreak of E. coli Infections Linked to Romaine Lettuce: Final Update. Available online at: https://www.cdc.gov/ecoli/2018/o157h7-11-18/index.html (accessed November 5, 2019).
Station, TX, USA. Research sponsor had no role in experimental design, data collection and analysis, manuscript preparation, journal selection, or manuscript submission.

\section{ACKNOWLEDGMENTS}

Authors acknowledge that physico-chemical data and microbiological data appear within the Ph.D. theses of authors Yegin (2020) and Perez-Lewis (2015), respectively, on file at the Texas A\&M University Libraries, College Station, TX, USA (library.tamu.edu).

CDC (2020). Reports of Seleted E. coli Outbreak Investigations. Available online at: https://www.cdc.gov/ecoli/outbreaks.html (accessed March 23, 2020).

Fatica, M. K., and Schneider, K. R. (2011). Salmonella and produce: survival in the plant environment and implications in food safety. Virulence 2, 573-579. doi: 10.4161/viru.2.6.17880

FDA (2020). Title 21, U.S. Code of Federal Regulations, \$112: Standards for the Growing, Harvesting, Packing, and Holding of Produce for Human Consumption. Available online at: https://www.ecfr.gov/cgi-bin/text-idx?SID= 6065be805bbe8488f8cf57bf0299dfd $9 \& \mathrm{mc}=$ true \&node=pt21.2.112\&rgn=div5 (accessed March 23, 2020).

FDA (2021). Inactive Ingredient Search for Approved Drug Products. Available online at: https://www.accessdata.fda.gov/scripts/cder/iig/index.cfm?event= browseByLetter.page\&Letter=P (accessed March 2, 2021).

Gaysinsky, S., Davidson, P. M., Bruce, B. D., and Weiss, J. (2005). Stability and antimicrobial efficiency of eugenol encapsulated in surfactant micelles as affected by temperature and pH. J. Food Protect. 68, 1359-1366. doi: 10.4315/0362-028X-68.7.1359

Gaysinsky, S., Davidson, P. M., McClements, D. J., and Weiss, J. (2008). Formulation and characterization of phytophenol-carrying antimicrobial microemulsions. Food Biophys. 3, 54-65. doi: 10.1007/s11483-0079048-1

Harris, L. J., Farber, J. N., Beuchat, L. R., Parish, M. E., Suslow, T. V., Garrett, E. H., et al. (2003). Outbreaks associated with fresh produce: Incidence, growth, and survival of pathogens in fresh and fresh-cut produce. Comp. Rev. Food Sci. Food Safety 2, 78-141. doi: 10.1111/j.1541-4337.2003.tb00031.x

Hill, L. E., Gomes, C., and Taylor, T. M. (2013). Characterization of beta-cyclodextrin inclusion complexes containing essential oils, transcinnamaldehyde, eugenol, cinnamon bark extract, and clove bud extracts for antimicrobial delivery applications. Food Sci. Technol. 51, 86-93. doi: 10.1016/j.lwt.2012.11.011

Hill, L. E., and Gomes, C. L. (2014). Optimization of synthesis process of thermally-responsive poly-n-isopropylacrylamide nanoparticles for controlled release of antimicrobial hydrophobic compounds. Mat. Res. Express 1:045404. doi: 10.1088/2053-1591/1/4/045404

Huang, K., and Nitin, N. (2019). Antimicrobial particle-based novel sanitizer for enhanced decontamination of fresh produce. Appl. Environ. Microbiol. 85, e02599-e02518. doi: 10.1128/AEM.02599-18

Kang, J.-H., and Song, K. B. (2018). Inhibitory effect of plant essential oil nanoemulsions against Listeria monocytogenes, Escherichia coli O157:H7, and Salmonella typhimurium on red mustard leaves. Innov. Food Sci. Emerg. Technol. 45, 447-454. doi: 10.1016/j.ifset.2017.09.019

Mora-Huertas, C. E., Fessi, H., and Elaissari, A. (2010). Polymerbased nanocapsules for drug delivery. Int. J. Pharm. 385, 113-142. doi: 10.1016/j.ijpharm.2009.10.018

Nowotarska, S. W., Nowotarski, K. J., Friedman, M., and Situ, C. (2014). Effect of structure on the interactions between five natural antimicrobial compounds and phospholipids of bacterial cell membrane on model monolayers. Molecules 19, 7497-7515. doi: 10.3390/molecules19067497

Painter, J. A., Hoekstra, R. M., Ayers, T., Tauxe, R. V., Braden, C. R., Angulo, F. J., et al. (2013). Attribution of foodborne illnesses, hospitalizations, and deaths 
to food commodities by using outbreak data, United States, 1998-2008. Emerg. Infect. Dis. 19, 407-415. doi: 10.3201/eid1903.111866

Pao, S., Long, W., Kim, C., and Rafie, A. R. (2012). Salmonella population rebound and its prevention on spray washed and non-washed jalapeño peppers and roma tomatoes in humid storage. Foodborne Path. Dis. 9, 361-366. doi: $10.1089 /$ fpd.2011.1051

Perez-Lewis, K. (2015). Novel Interventions for Reducing Pathogen Attachment and Growth on Fresh Produce. [dissertation]. (College Station, TX: Texas A\&M University).

Perez-Lewis, K. L., Yegin, Y., Cisneros-Zevallos, L., Castillo, A., Kerth, C. R., Akbulut, M., et al. (2018). Geraniol-loaded polymeric nanoparticles inhibit enteric pathogens on spinach during posttreatment refrigerated and temperature abuse storage. Front. Sustain. Food Syst. 2:16. doi: 10.3389 /fsufs. 2018.00004

Pham Trong, L. C., Djabourov, M., and Ponton, A. (2008). Mechanisms of micellization and rheology of PEO-PPO-PEO triblock copolymers with various architectures. J. Colloid Interface Sci. 328, 278-287. doi: 10.1016/j.jcis.2008.09.029

Prud'homme, R. K., Wu, G., and Schneider, D. K. (1996). Structure and rheology studies of poly(oxyethylene-oxypropylene-oxyethylene) aqueous solution. Langmuir 12, 4651-4659. doi: 10.1021/la951506b

Ruengvisesh, S., Kerth, C. R., and Taylor, T. M. (2019a). Inhibition of Escherichia coli $\mathrm{O} 157: \mathrm{H} 7$ and Salmonella enterica isolates on spinach leaf surfaces using eugenol-loaded surfactant micelles. Foods 8:575. doi: 10.3390/foods8110575

Ruengvisesh, S., Loquercio, A., Castell-Perez, E., and Taylor, T. M. (2015). Inhibition of bacterial pathogens in medium and on spinach leaf surfaces using plant-derived antimicrobials loaded in surfactant micelles. J. Food Sci. 80, M2522-M2529. doi: 10.1111/1750-3841.13085

Ruengvisesh, S., Oh, J. K., Kerth, C. R., Akbulut, M., and Taylor, T. M. (2019b). Inhibition of bacterial human pathogens on tomato skin surfaces using eugenol-loaded surfactant micelles during refrigerated and abuse storage. J. Food Saf. 39:e12598. doi: 10.1111/jfs. 12598

Scallan, E., Hoekstra, R. M., Angulo, F. J., Tauxe, R. V., Widdowson, M. A., Roy, S. L., et al. (2011). Foodborne illness acquired in the United States-major pathogens. Emerg. Infect. Dis. 17, 7-15. doi: 10.3201/eid1701.P11101

Singh, P., Hung, Y.-C., and Qi, H. (2018). Efficacy of peracetic acid in inactivating foodborne pathogens on fresh produce surface. J. Food Sci. 83, M432-M439. doi: 10.1111/1750-3841.14028

Smolinski, H. S., Wang, S., Ren, L., Chen, Y., Kowalcyk, B., Thomas, E., et al. (2018). Transfer and redistribution of Salmonella Typhimurium LT2 and Escherichia coli O157:H7 during pilot-scale processing of baby spinach, cilantro, and romaine lettuce. J. Food Prot. 81, 953-962. doi: 10.4315/0362-028X.JFP-17-420

Walsh, K. A., Bennett, S. D., Mahovic, M., and Gould, L. H. (2014). Outbreaks associated with cantaloupe, watermelon, and honeydew in the United States, 1973-2011. Foodborne Path. Dis. 11, 945-952. doi: 10.1089/fpd.2014.1812
Wanka, G., Hoffman, H., and Ulbricht, W. (1994). Phase diagrams and aggregation behavior of poly(oxyethylene)-poly(oxypropylene)-poly(oxyethylene) triblock copolymers in aqueous solutions. Macromolecules 27, 4145-4159. doi: 10.1021/ma00093a016

Wuang, S. C., Neoh, K. G., Kang, E.-T., Leckband, D. E., and Pack, D. W. (2011). Acid-sensitive magnetic nanoparticles as potential drug depots. AIChE J. 57, 1638-1645. doi: 10.1002/aic. 12373

Yegin, Y. (2020). Mechanistic Investigation of Inactivating Bacterial Food Pathogens With Nanoparticles. [dissertation]. (College Station, TX: Texas A\&M University).

Yegin, Y., Perez-Lewis, K. L., Zhang, M., Akbulut, M., and Taylor, T. M. (2016). Development and characterization of geraniol-loaded polymeric nanoparticles with antimicrobial activity against foodborne bacterial pathogens. J. Food Eng. 170, 64-71. doi: 10.1016/j.jfoodeng.2015.09.017

Yoon, J.-H., and Lee, S.-Y. (2018). Review: comparison of the effectiveness of decontaminating strategies for fresh fruits and vegetables and related limitations. Crit. Rev. Food Sci. Nutr. 58, 3189-3208. doi: 10.1080/10408398.2017.1354813

Yossa, N., Patel, J., Millner, P., and Lo, Y. M. (2012). Essential oils reduce Escherichia coli O157:H7 and Salmonella on spinach leaves. J. Food Prot. 75, 488-496. doi: 10.4315/0362-028X.JFP-11-344

Yossa, N., Patel, J., Millner, P., Ravishankar, S., and Lo, Y. M. (2013). Antimicrobial activity of plant essential oils against Escherichia coli O157:H7 and Salmonella on lettuce. Foodborne Path. Dis. 10, 87-96. doi: 10.1089/fpd.201 2.1301

Yun, J., Yan, R., Fan, X., Gurtler, J., and Phillips, J. (2013). Fate of E. coli O157:H7, Salmonella spp. and potential surrogate bacteria on apricot fruit, following exposure to UV-C light. Int. J. Food Microbiol. 166, 356-363. doi: 10.1016/j.ijfoodmicro.2013. 07.021

Zhu, L., Olsen, C., McHugh, T., Friedman, M., Levin, C. E., Jaroni, D., et al. (2020). Edible films containing carvacrol and cinnamaldehyde inactivate Escherichia coli $\mathrm{O} 157: \mathrm{H7}$ on organic leafy greens in sealed plastic bags. J. Food Safety 8:12758. doi: $10.1111 /$ jfs. 12758

Conflict of Interest: The authors declare that the research was conducted in the absence of any commercial or financial relationships that could be construed as a potential conflict of interest.

Copyright (C) 2021 Yegin, Perez-Lewis, Liu, Kerth, Cisneros-Zevallos, Castillo, Akbulut and Taylor. This is an open-access article distributed under the terms of the Creative Commons Attribution License (CC BY). The use, distribution or reproduction in other forums is permitted, provided the original author $(s)$ and the copyright owner(s) are credited and that the original publication in this journal is cited, in accordance with accepted academic practice. No use, distribution or reproduction is permitted which does not comply with these terms. 